Louisiana State University

LSU Digital Commons

Faculty Publications

Department of Mathematics

8-1-2016

\title{
Reduction model approach for linear time-varying systems with input delays based on extensions of Floquet theory
}

\author{
Frédéric Mazenc \\ Laboratoire des Signaux et Systèmes \\ Michael Malisoff \\ Louisiana State University
}

Follow this and additional works at: https://digitalcommons.Isu.edu/mathematics_pubs

\section{Recommended Citation}

Mazenc, F., \& Malisoff, M. (2016). Reduction model approach for linear time-varying systems with input delays based on extensions of Floquet theory. Systems and Control Letters, 94, 70-76. https://doi.org/ 10.1016/j.sysconle.2016.05.013

This Article is brought to you for free and open access by the Department of Mathematics at LSU Digital Commons. It has been accepted for inclusion in Faculty Publications by an authorized administrator of LSU Digital Commons. For more information, please contact ir@lsu.edu. 


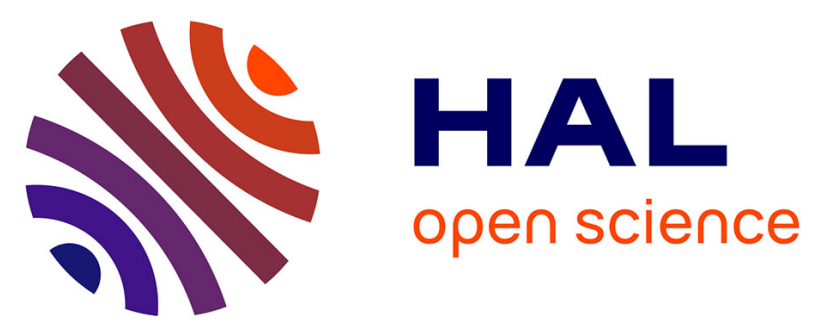

\section{Reduction model approach for linear time-varying systems with input delays based on extensions of Floquet theory}

Frédéric Mazenc, Michael Malisoff

\section{- To cite this version:}

Frédéric Mazenc, Michael Malisoff. Reduction model approach for linear time-varying systems with input delays based on extensions of Floquet theory. Systems and Control Letters, Elsevier, 2016, 94 (August 2016), pp.70-76. 10.1016/j.sysconle.2016.05.013 . hal-01389860

\section{HAL Id: hal-01389860 \\ https://hal.inria.fr/hal-01389860}

Submitted on 30 Oct 2016

HAL is a multi-disciplinary open access archive for the deposit and dissemination of scientific research documents, whether they are published or not. The documents may come from teaching and research institutions in France or abroad, or from public or private research centers.
L'archive ouverte pluridisciplinaire HAL, est destinée au dépôt et à la diffusion de documents scientifiques de niveau recherche, publiés ou non, émanant des établissements d'enseignement et de recherche français ou étrangers, des laboratoires publics ou privés. 


\title{
Reduction Model Approach for Linear Time-Varying Systems with Input Delays based on Extensions of Floquet Theory *
}

\author{
Frédéric Mazenc $^{\dagger} \quad$ Michael Malisoff $\ddagger$
}

December 31, 2015

\begin{abstract}
We solve stabilization problems for linear time-varying systems under input delays. We show how changes of coordinates lead to systems with time invariant drifts, which are covered by the reduction model method and which lead to the problem of stabilizing a time-varying system without delay. For continuous time periodic systems, we can use Floquet theory to find the changes of coordinates. We also prove an analogue for discrete time systems, through a discrete time extension of Floquet theory.
\end{abstract}

\section{Introduction}

This note continues our search (begun in [22] and [24]) for extensions of the classical reduction model method that cover time-varying systems with input delays. Input delays are common when controllers are remotely implemented; see $[4,7,8,17,18,30]$ for more motivation. The reduction method has its origins in the works [2], [19], and [21] by Artstein and others, who focused on continuous time time-invariant linear systems.

Stabilization problems for linear time-varying systems with delays have been studied in fewer works. In most of them, time-varying Lyapunov functions are needed; see for instance [1] and [27] for the use of strict Lyapunov functions, and [29] for Razumikhin-Lyapunov functions. One useful Lyapunov-based approach to delay systems entails solving the stabilization problem with the input delay set equal to zero, and then using Lyapunov-Krasovskii functionals to look for upper bounds on the input delays that the closed loop system can tolerate without sacrificing the stability performance; see [20, 23]. Linear time-varying systems arise in the context of the local stabilization of a trajectory of a nonlinear system, but are beyond the scope of the classical reduction model method. The main differences between reduction approaches and the Lyapunov-Krasovskii functional approaches such as those in [23] are that (a) under certain delay bounds, methods such as [23] lead to relatively simple controllers that do not require the distributed terms that are used in reduction model methods and (b) reduction model methods usually make it possible to compensate for arbitrarily long input delays, by using the delay value in the dynamic feedback control design.

Our work [24] extends the reduction model method to linear time-varying systems, using two approaches. One approach in [24] leads to a control formula that involves the fundamental matrix for the corresponding uncontrolled system (i.e., the time-varying system obtained from the original system by setting the input equal to zero in the original system), and so may be difficult to apply in practice. The other control design in [24] does not require a formula for the fundamental matrix, but requires that the input delay stay below a suitable constant bound. By contrast, [22] covers time-varying nonlinear systems whose nonlinear parts satisfy certain conditions, and then builds a reduction model control for the linearization of the system, and finally shows local stability of the original nonlinear system in closed loop with the reduction model control.

One natural research direction for addressing the challenges of extending the reduction model method to time-varying linear systems, as well as analogous problems for discrete time systems, is to seek analogues

${ }^{*}$ Key Words: delay, time-varying, Floquet theory, reduction model. Corresponding Author: Frédéric Mazenc, Tel.: + 33 [0] 1698517 62, Mobile: 06070423 52, Fax: + 33 [0] 169851765.

${ }^{\dagger}$ Mazenc is with EPI DISCO INRIA-Saclay, L2S, CNRS CentraleSupelec, 3 rue Joliot Curie, 91192 Gif-sur-Yvette, France (email: frederic.mazenc@12s.centralesupelec.fr).

${ }_{\ddagger}^{\ddagger}$ Malisoff is with Department of Mathematics, 303 Lockett Hall, Louisiana State University, Baton Rouge, LA 70803-4918, USA (email: malisoff@lsu.edu). Supported by NSF Division of Electrical, Communications and Cyber Systems Grant 1102348. 
of Floquet's theory; see [26, Section 3.5] for the basic results of Floquet theory. Floquet's theory covers systems without controls. One of its basic results is that if a time-varying linear system is periodic, then it can be transformed into a time-invariant system through a periodic change of coordinates. This suggests the possibility of using Floquet theory to transform a time-varying linear control system into a new time-varying linear control system with a time-invariant draft, and then stabilizing the new system using the reduction approach. One of the key observations in this work is that such a transformation can indeed be done under periodicity of the coefficient matrices in the system, and that this simplifies the stabilization problem to one that involves globally asymptotically stabilizing systems with no delay. Our assumptions are novel as well. We also provide analogues for nonperiodic or discrete time systems.

Discrete time systems with delay are important because they can be used to model some engineering devices; see [6], [9], [11], and [14]. However, not many contributions are concerned with time-varying discrete time systems with delay. Our discrete time delayed systems in this work have the form

$$
x_{k+1}=A_{k} x_{k}+B_{k} u_{k-r}
$$

where $x_{k} \in \mathbb{R}^{n}$ is the state, $u_{k} \in \mathbb{R}^{p}$ is the control, and $r \in \mathbb{N}$ is the delay. Here and in the sequel, the dimensions and delays are arbitrary. For the case of time-invariant coefficients, the work [9] uses dynamic extensions to transform systems of the form (1) into systems with no delay, in the special case of networked control systems. See also [12] for a prediction based approach for (1) in the time-invariant case. For timevarying continuous time systems with delay in the input, the reduction model approach can be applied under conditions pertaining to the speed of variation of the time-varying matrices; see [24]. However, to the best of our knowledge, no discrete time version of [24] exists. Also, [16] is concerned with time-invariant systems.

We propose a rather general solution to the problem of exponential stabilization of (1) through the reduction model approach, including cases where the coefficient matrices are not necessarily periodic, with an arbitrarily large delay $r$. It decomposes into two steps. First, under reasonable assumptions, we transform (1) into a system that is autonomous when the control is set equal to zero. Then, we adapt the reduction method to the resulting dynamics, using a novel discrete time analogue of an operator that is used in the predictor based analysis in [16]. Our treatment of (1) also has implications for using reduction model controllers in continuous time systems, because in practice, implementing controllers in continuous time systems uses discretizations, leading to discrete time delay systems of the form (1). We illustrate our theory in two examples, including a discrete time linear system in which the coefficient matrices are not periodic.

\section{Preliminary Results in Continuous Time}

We use the following notation and definitions. We let $|\cdot|$ denote the usual Euclidean norm of matrices and vectors, and $I_{n}$ is the $n \times n$ identity matrix. Here and in the sequel, the dimensions of our Euclidean spaces are arbitrary unless other indicated. Given any function $\phi: S \rightarrow \mathbb{R}^{p}$ that is defined on any subset $S$ of a Euclidean space, denote its supremum over any set $J \subseteq S$ by $|\phi|_{J}$. We set $\mathbb{N}=\{1,2, \ldots\}$. We often leave out the arguments of functions, when they are clear from the context.

\subsection{Fundamental General Result}

Consider the system

$$
\dot{x}=A(t) x+F(t) u(t-\tau)
$$

where the state $x$ and the input $u$ are valued in $\mathbb{R}^{n}$ and $\mathbb{R}^{p}$ respectively, the functions $A: \mathbb{R} \rightarrow \mathbb{R}^{n \times n}$ and $F: \mathbb{R} \rightarrow \mathbb{R}^{n \times p}$ are continuous, and $\tau>0$ is any positive constant delay. We introduce the following assumption. See below for ways to build the required function $P$.

Assumption 1. There exist a constant matrix $A_{c} \in \mathbb{R}^{n \times n}$, a $C^{1}$ function $P: \mathbb{R} \rightarrow \mathbb{R}^{n \times n}$ such that $P^{-1}(t)$ is defined for all $t$, and a constant $p_{M}>0$ such that

$$
|P(t)|+\left|P^{-1}(t)\right| \leq p_{M}
$$

and

$$
\dot{P}(t)=A_{c} P(t)-P(t) A(t) .
$$

hold for all $t \geq 0$. 
We use the following key observations:

Lemma 1. Assume that the system (2) satisfies Assumption 1. Then the time-varying change of coordinates

$$
z=P(t) x
$$

transforms (2) into

$$
\dot{z}(t)=A_{c} z(t)+P(t) F(t) u(t-\tau)
$$

Also, the operator

$$
Z(t)=z(t)+\int_{t-\tau}^{t} e^{A_{c}(t-m-\tau)} P(m+\tau) F(m+\tau) u(m) \mathrm{d} m
$$

transforms (6) into the system

$$
\dot{Z}(t)=A_{c} Z(t)+e^{-A_{c} \tau} P(t+\tau) F(t+\tau) u(t)
$$

with the state variable $Z$.

Proof. Our choice (5) of $z$ gives

$$
\dot{z}(t)=\dot{P}(t) x(t)+P(t) \dot{x}(t)=\dot{P}(t) x(t)+P(t)[A(t) x(t)+F(t) u(t-\tau)] .
$$

Then (6) follows from (4) and our choice of $z$. Also, the time derivative of (7) along all solutions of (6) is

$$
\begin{aligned}
\dot{Z}(t)= & A_{c} z(t)+P(t) F(t) u(t-\tau)+A_{c} \int_{t-\tau}^{t} e^{A_{c}(t-m-\tau)} P(m+\tau) F(m+\tau) u(m) \mathrm{d} m \\
& +e^{-A_{c} \tau} P(t+\tau) F(t+\tau) u(t)-P(t) F(t) u(t-\tau) \\
= & A_{c} Z(t)+e^{-A_{c} \tau} P(t+\tau) F(t+\tau) u(t)
\end{aligned}
$$

which gives the second conclusion.

The message of Lemma 1 is that if one knows a function $P$ that leads to a time-invariant system when the input is set to zero, then in practice, we can use the reduction model approach to obtain a new system without delays. We study the problem of finding $P$ in the next subsection.

Remark 1. Before discussing ways to find $P$, we remark that similar reasoning applies to systems

$$
\dot{x}=A(t) x+\int_{t-\tau}^{t} F(\ell) u(\ell) \mathrm{d} \ell
$$

with distributed delay in the input and continuous matrix valued functions $A$ and $F$. To see how, notice that if Assumption 1 is satisfied, and if we define $z$ by (5) as before and redefine $Z$ to be

$$
Z(t)=z(t)+\int_{t-\tau}^{t} e^{A_{c}(t-m-\tau)} P(m+\tau)\left(\int_{m}^{t} F(\ell) u(\ell) \mathrm{d} \ell\right) \mathrm{d} m
$$

then similar reasoning to the proof of Lemma 1 gives

$$
\dot{z}(t)=A_{c} z(t)+P(t) \int_{t-\tau}^{t} F(\ell) u(\ell) \mathrm{d} \ell
$$

and then

$$
\dot{Z}(t)=A_{c} Z(t)+\left(\int_{t-\tau}^{t} e^{A_{c}(t-\ell-\tau)} P(\ell+\tau) \mathrm{d} \ell\right) F(t) u(t)
$$

so we eliminated the distributed delay.

\subsection{Particular Case}

In order to get a function $P$ for which Assumption 1 is satisfied, we impose checkable conditions on the corresponding zero input subsystem

$$
\dot{x}=A(t) x
$$

which we later show how to verify using Floquet theory when $A$ is periodic. We introduce the following assumption: 
Assumption 2. All solutions of the system (15) are of the form

$$
x(t)=K(t) E(t) x(0),
$$

where $K: \mathbb{R} \rightarrow \mathbb{R}^{n \times n}$ and $E: \mathbb{R} \rightarrow \mathbb{R}^{n \times n}$ are everywhere invertible functions of class $C^{1}, K^{-1}$ is of class $C^{1}$, and $E$ is such that there exists a constant matrix $E_{c}$ such that

$$
\dot{E}(t) E^{-1}(t)=E_{c}
$$

holds for all $t \geq 0$. Moreover, there is a constant $k_{c}>0$ such that

$$
|K(t)|+\left|K^{-1}(t)\right| \leq k_{c}
$$

for all $t \geq 0$.

We can often find functions $E$ satisfying the preceding requirements. For example, they hold if $E(t)=$ $e^{M t} G$, where $M \in \mathbb{R}^{n \times n}$ and $G \in \mathbb{R}^{n \times n}$ are constant matrices and $G$ is invertible. Indeed, in this case $\dot{E}(t)=M e^{M t} G, E^{-1}(t)=G^{-1} e^{-M t}$ and therefore also $\dot{E}(t) E^{-1}(t)=M$ are satisfied. See below for cases where Assumption 2 is satisfied. We are ready to state and prove the following result:

Lemma 2. Let the system (2) be such that (15) satisfies Assumption 2. Then the change of coordinates

$$
z=L(t) x
$$

with $L=K^{-1}$ gives the new system

$$
\dot{z}(t)=E_{c} z(t)+L(t) F(t) u(t-\tau)
$$

with a time invariant drift term.

Proof. From (15) and (16), it follows that

$$
\dot{x}(t)=A(t) K(t) E(t) x(0)
$$

and

$$
\dot{x}(t)=\dot{K}(t) E(t) x(0)+K(t) \dot{E}(t) x(0)
$$

hold for all $x(0) \in \mathbb{R}^{n}$. This gives

$$
A(t) K(t) E(t)=\dot{K}(t) E(t)+K(t) \dot{E}(t) .
$$

Here and in the sequel, all equalities or inequalities are to be understood to hold for all $t \geq 0$. Since $E$ is invertible everywhere, (23) gives $\dot{K}(t)+K(t) \dot{E}(t) E^{-1}(t)=A(t) K(t)$. From (17), it follows that $\dot{K}(t)+K(t) E_{c}=A(t) K(t)$. This gives

$$
L(t) \dot{K}(t) L(t)+E_{c} L(t)=L(t) A(t) .
$$

Since $L(t) K(t)=I_{n}$ for all $t$, it follows that $L(t) \dot{K}(t)+\dot{L}(t) K(t)=0$. Therefore, $(24)$ gives $-\dot{L}(t) K(t) L(t)+$ $E_{c} L(t)=L(t) A(t)$. We deduce that $\dot{L}(t)=E_{c} L(t)-L(t) A(t)$. This equality and (18) ensure that Assumption 1 is satisfied with $L=P$ and $E_{c}=A_{c}$. Therefore Lemma 1 applies, which proves Lemma 2.

We next turn to our general ways to find functions $K$ and $E$ that satisfy Assumption 2.

\section{Results Based on Floquet's Theory in Continuous Time}

\subsection{Theoretical result}

In the particular case where the function $A$ is periodic, Floquet's theory (e.g., [26, Section 3.5]) guarantees that there are two functions $K$ and $E$ such that Assumption 2 is satisfied. More precisely, thanks to Floquet's theory, we can establish the following result: 
Theorem 1. Consider the system (2) and assume that $A$ is periodic of any period $T>0$. Then there exist an everywhere invertible $C^{1}$ function $H: \mathbb{R} \rightarrow \mathbb{R}^{n \times n}$ that is periodic of period $T$ and a constant matrix $R \in \mathbb{R}^{n \times n}$ such that the change of coordinates $z=H(t) x$ transforms (2) into

$$
\dot{z}(t)=R z(t)+H(t) F(t) u(t-\tau)
$$

for all $t \geq 0$.

Proof. Consider the system $\dot{x}=A(t) x$ from (15) associated with the system (2). Let $X: \mathbb{R} \rightarrow \mathbb{R}^{n \times n}$ be the fundamental solution of (15) such that $X(0)=I_{n}$, so $\dot{X}(t)=A(t) X(t)$ and $X(t)$ is invertible for all $t \in \mathbb{R}$. Then Floquet's theory (e.g., from [26, Section 3.5]) ensures that there exist a constant matrix $R \in \mathbb{R}^{n \times n}$, and an everywhere invertible $C^{1}$ function $Q: \mathbb{R} \rightarrow \mathbb{R}^{n \times n}$ that is periodic of period $T$, such that

$$
X(t)=Q(t) e^{R t}
$$

holds for all $t \in \mathbb{R}$. Then $Q^{-1}$ is $C^{1}$, because $X$ is $C^{1}$ and invertible. By (26), we deduce that Assumption 2 is satisfied with $K(t)=Q(t), E(t)=e^{R t}$, and $E_{c}=R$. It follows from Lemma 2 that the change of coordinates

$$
z(t)=Q^{-1}(t) x(t)
$$

transforms the system (2) into $\dot{z}(t)=R z(t)+Q^{-1}(t) F(t) u(t-\tau)$. Since $Q^{-1}$ is periodic of period $T$, this allows us to conclude by setting $H=Q^{-1}$.

A challenge in applying Theorem 1 is that determining the function $Q$ is not easy. This fact is a fundamental limitation of Floquet's theory. This remark motivates the next subsection.

\subsection{More Applied Results Based on Floquet's Theory}

We again consider the system (15) in the case where $A$ is periodic of some period $T$. Then the decomposition (26) of the fundamental matrix gives $I_{n}=X(0)=Q(0)=Q(T)$, so $Q(T)=I_{n}$ and $X(T)=e^{R T}$. Through numerical simulations, we can determine $X(T)$, which is invertible. Recalling from [10] that an invertible real matrix has a real matrix logarithm (denoted by $\log$ ) if and only if each Jordan block in its Jordan decomposition corresponding to a negative eigenvalue occurs an even number of times, it follows that if $X(T)$ has no eigenvalues that are contained in $(-\infty, 0)$, then we can set $R=\frac{1}{T} \log (X(T))$; see [15, Section 1.6] for the definition of and formulas for matrix logarithms of invertible matrices.

Next, we return to (2). Let us show how we can determine a function $Q$ such that $X(t)=Q(t) e^{R t}$, which makes it possible to apply Theorem 1 in practice. Since $X(t)=Q(t) e^{R t}$ is a fundamental solution of $\dot{x}=A(t) x$, we get $\dot{X}(t)=\dot{Q}(t) e^{R t}+Q(t) R e^{R t}=A(t) Q(t) e^{R t}$, so cancelling $e^{R t}$ from both sides gives $Q^{-1}(t) \dot{Q}(t) Q^{-1}(t)+R Q^{-1}(t)=Q^{-1}(t) A(t)$. This gives

$$
R H(t)-H(t) A(t)=-Q^{-1} \dot{Q}(t) Q^{-1}(t)=\dot{H}(t),
$$

where $H=Q^{-1}$, and where the last equality follows by computing the derivative of $Q(t) Q^{-1}(t)$ using the product rule. Therefore, we consider the system (2) with the dynamic extension $\dot{\varpi}(t)=R \varpi(t)-\varpi(t) A(t)$. Then, since $Q(0)=I_{n}$, it follows that when $\varpi(0)=I_{n}$, we have $H=\varpi$. Hence, through a simple dynamic extension with the identity as the initial condition, we have $H$.

Then Theorem 1 implies that the change of coordinates $z(t)=H(t) x(t)$ gives

$$
\dot{z}(t)=R z(t)+\varpi(t) F(t) u(t-\tau) .
$$

Therefore, when we apply the reduction model approach, we consider the operator $Z$ from (7), namely,

$$
Z(t)=z(t)+\int_{t-\tau}^{t} e^{R(t-m-\tau)} \varpi(m+\tau) F(m+\tau) u(m) \mathrm{d} m .
$$

Although this involves future values of $\varpi$, the function $\varpi$ is periodic of period $T$. Therefore, we can replace $\varpi(m+\tau)$ by $\varpi(m+\tau-k T)$, where $k$ is a sufficiently large integer. After such a substitution, (30) is only valid for sufficiently large $t$. Then the reasoning that gave the delay free system (8) gives

$$
\dot{Z}(t)=R Z(t)+e^{-R \tau} \varpi(t+\tau-k T) F(t+\tau) u(t) .
$$


This means that if we stabilize this system using a state feedback, it will depend on the past $\varpi$ values. Alternatively, we can consider the system

$$
\left\{\begin{aligned}
\dot{x}(t) & =A(t) x(t)+F(t) u(t-\tau) \\
\dot{\varpi}(t) & =R \varpi(t)-\varpi(t) A(t) \\
\varpi(l T) & =I_{n}, l \in\{0,1,2, \ldots\}
\end{aligned}\right.
$$

to obtain the closed loop system.

Remark 2. If we consider the system $\dot{x}(t)=f(x(t))$ with $x$ valued in $\mathbb{R}^{2}$ with a periodic solution $r(t)$ of some period $T$ and with $\dot{r}(0) \neq 0$ and evaluate the linear approximation around this solution, then for the corresponding linearized system $\dot{x}=A(t) x$ (where $A(t)$ is the Jacobian matrix $A(t)=D f(r(t))$ ), we know that 1 is one of the eigenvalues of the corresponding matrix $e^{R T}$. This follows because $\dot{r}(t)$ is a solution of $\dot{x}=A(t) x$, so $\dot{r}(0)=\dot{r}(T)=Q(T) e^{R T} \dot{r}(0)=e^{R T} \dot{r}(0)$. Then, using the relation

$$
\rho_{1} \rho_{2}=\exp \left(\int_{0}^{T} \operatorname{trace}(A(\ell)) \mathrm{d} \ell\right)
$$

between the eigenvalues $\rho_{1}$ and $\rho_{2}$ of $e^{R T}$ that is provided by Floquet's theory (e.g., [3, Proposition 2.39]), we get $\rho_{1}=1$ and

$$
\rho_{2}=\exp \left(\int_{0}^{T} \operatorname{trace}(A(\ell)) \mathrm{d} \ell\right) .
$$

Therefore one does not need to use a computer to get the two eigenvalues of $e^{R T}$ to compute the matrix logarithm for the Floquet theory decomposition of the fundamental matrix.

Before illustrating our theory in the continuous time case in Section 6, we provide the following discrete time analogues of the preceding results.

\section{Preliminary Results in Discrete Time}

We propose a more general version of Floquet's theory for discrete time systems than what was developed in [13] and [28]. We wish to design delay compensating stabilizing controllers for systems of the form

$$
x_{k+1}=A_{k} x_{k}+B_{k} u_{k-r}
$$

where $x_{k} \in \mathbb{R}^{n}$ is the state, $u_{k} \in \mathbb{R}^{p}$ is the control, and $r \in \mathbb{N}$ is the delay. We first consider the system

$$
x_{k+1}=A_{k} x_{k}
$$

with $k \in \mathbb{N}$ and $x_{k}$ valued in $\mathbb{R}^{n}$. Since our extension does not require $A_{k}$ to be periodic, we believe that it is new. Moreover, our changes of coordinates are explicit in many interesting cases. The work [28] shows that in the periodic context, matrices similar to the $R_{k}$ 's defined below can be found, but in [13], the change of coordinates is not explicit, and $[13,28]$ do not cover reduction model controls. We introduce two assumptions.

Assumption 3. For all $k \geq 0$, the matrix $A_{k}$ is invertible.

Assumption 3 allows us to introduce a sequence of matrices defined by

$$
P_{k}=\prod_{i=0}^{k-1} A_{i}^{-1}
$$

for all $k \geq 1$ and $P_{0}=I_{n}$. Then $P_{k}$ is invertible for all $k \in \mathbb{N}$. Our second assumption is:

Assumption 4. There are an invertible matrix $A_{*} \in \mathbb{R}^{n \times n}$ and a real number $c>0$ such that the sequence

$$
R_{k}=A_{*}^{k} P_{k}
$$

satisfies

$$
\left|R_{k}^{-1}\right|+\left|R_{k}\right| \leq c
$$

for all $k \in \mathbb{N}$. 
We prove the following:

Lemma 3. Let Assumptions 3-4 hold. Then for each solution sequence $x_{k}$ of (36), the sequence

$$
z_{k}=R_{k} x_{k}
$$

satisfies

$$
z_{k+1}=A_{*} z_{k}
$$

for all $k$.

Proof. We have $z_{k+1}=R_{k+1} A_{k} x_{k}=R_{k+1} A_{k} R_{k}^{-1} z_{k}$. Here and in the sequel, all equalities or inequalities should be understood to hold for all integers $k \geq 0$, unless otherwise indicated. Our definitions give

$$
R_{k+1} A_{k} R_{k}^{-1}=A_{*}^{k+1}\left(\prod_{i=0}^{k} A_{i}^{-1}\right) A_{k} P_{k}^{-1}\left(A_{*}^{k}\right)^{-1}=A_{*}^{k+1}\left(\prod_{i=0}^{k-1} A_{i}^{-1}\right) P_{k}^{-1}\left(A_{*}^{k}\right)^{-1}=A_{*} .
$$

By right multiplying through (42) by $z_{k}$, it follows that $z_{k}$ is solution of (41), which proves the lemma.

If $A_{*}$ is known, then $R_{k}$ is given by an explicit formula. Finding $A_{*}$ can be difficult. However, if $A_{k}$ is periodic with some period $p$, and if $A_{p-1} A_{p-2} \ldots A_{0}$ has no eigenvalues that lie in the interval $(-\infty, 0]$, then [5] and [15, Theorem 7.2] provide a matrix $A_{*} \in \mathbb{R}^{n \times n}$ such that $A_{*}^{p}=A_{p-1} A_{p-2} \ldots A_{0}$, as well as algorithms for computing this $p$ th root matrix. Since (42) gives $R_{k+1}=A_{*} R_{k} A_{k}^{-1}$ and $R_{1}=A_{*} A_{0}^{-1}$, we get $R_{k}=A_{*}^{k} A_{0}^{-1} A_{1}^{-1} \ldots A_{k-1}^{-1}$ for all $k \geq 1$, which gives $R_{p}=I_{n}$ and periodicity of $R_{k}$. Hence, this $A_{*}$ satisfies Assumption 4. To check that this $p$ th root matrix is invertible, notice that if $v \in \mathbb{R}^{n} \backslash\{0\}$ were in the null space of $A_{*}$, then $0=A_{*}^{p} v=A_{p-1}\left(A_{p-2} \ldots A_{0} v\right)$, which contradicts the invertibility of the $A_{k}$ 's. See Section 6 for a nonperiodic example where we find $A_{*}$.

\section{Model Reduction in Discrete Time}

\subsection{Main Result}

In this section, we use Lemma 3 to extend the reduction model approach to a general family of systems of the type (35). We introduce the following assumption, which is a natural analogue of the usual requirement of controllable pairs of coefficient matrices, generalized to time-varying systems with delays:

Assumption 5. The system (35) is such that the sequence $A_{k}$ satisfies Assumptions 3-4, and the sequence $B_{k}$ is bounded. Also, there is a sequence $K_{k}$ such that the sequence $K_{k} R_{k}^{-1}$ is bounded and such that the system

$$
\zeta_{k+1}=H_{k} \zeta_{k}, \text { where } H_{k}=A_{k}+R_{k+1}^{-1} R_{k+r+1} B_{k+r} K_{k}
$$

is uniformly exponentially stable, where $R_{k}$ is the sequence defined in (38).

We prove the following:

Theorem 2. Assume that the system (35) satisfies Assumption 5. Then we can find positive constants $\bar{c}_{1}$ and $\bar{c}_{2}$ such that all trajectories of (35), in closed loop with

$$
u_{k}=K_{k} R_{k}^{-1}\left(A_{*}^{r} R_{k} x_{k}+\sum_{i=1}^{r} A_{*}^{i-1} R_{k+r-i+1} B_{k+r-i} u_{k-i}\right),
$$

satisfy $\left|x_{k}\right| \leq \bar{c}_{1} e^{\bar{c}_{2}\left(k_{0}-k\right)}\left(\left|x_{k_{0}}\right|+|u|_{\left[k_{0}-r, k_{0}\right]}\right)$ for all integers $k_{0} \geq 1$ and $k \geq k_{0}$.

Proof. We can use (42) to show that the change of coordinates $z_{k}=R_{k} x_{k}$ applied to (35) gives

$$
z_{k+1}=A_{*} z_{k}+G_{k} u_{k-r},
$$

where $G_{k}=R_{k+1} B_{k}$. Since $A_{*}$ is time-invariant, we can adapt ideas from [12] to the problem of stabilizing the system (45), as follows. 
We use the operator

$$
\Gamma_{k}=A_{*}^{r} z_{k}+\sum_{i=1}^{r} A_{*}^{i-1} G_{k+r-i} u_{k-i}
$$

Then,

$$
\begin{aligned}
\Gamma_{k+1} & =A_{*}^{r}\left(A_{*} z_{k}+G_{k} u_{k-r}\right)+\sum_{i=1}^{r} A_{*}^{i-1} G_{k+1+r-i} u_{k+1-i} \\
& =A_{*}^{r+1} z_{k}+A_{*}^{r} G_{k} u_{k-r}+\sum_{i=2}^{r} A_{*}^{i-1} G_{k+1+r-i} u_{k+1-i}+G_{k+r} u_{k} \\
& =A_{*}^{r+1} z_{k}+\sum_{i=2}^{r+1} A_{*}^{i-1} G_{k+1+r-i} u_{k+1-i}+G_{k+r} u_{k} .
\end{aligned}
$$

Using the definition of $\Gamma_{k}$, we get

$$
\begin{aligned}
\Gamma_{k+1} & =A_{*}\left(\Gamma_{k}-\sum_{i=1}^{r} A_{*}^{i-1} G_{k+r-i} u_{k-i}\right)+\sum_{i=2}^{r+1} A_{*}^{i-1} G_{k+1+r-i} u_{k+1-i}+G_{k+r} u_{k} \\
& =A_{*} \Gamma_{k}-\sum_{i=1}^{r} A_{*}^{i} G_{k+r-i} u_{k-i}+\sum_{i=1}^{r} A_{*}^{i} G_{k+r-i} u_{k-i}+G_{k+r} u_{k}=A_{*} \Gamma_{k}+G_{k+r} u_{k} .
\end{aligned}
$$

Then we can choose $u_{k}=K_{k} R_{k}^{-1} \Gamma_{k}$. This feedback is equivalent to the feedback in (44). Then (42) gives

$$
\begin{aligned}
\Gamma_{k+1} & =\left(A_{*}+G_{k+r} K_{k} R_{k}^{-1}\right) \Gamma_{k} \\
& =\left(R_{k+1} A_{k} R_{k}^{-1}+G_{k+r} K_{k} R_{k}^{-1}\right) \Gamma_{k} \\
& =R_{k+1}\left(A_{k}+R_{k+1}^{-1} R_{k+r+1} B_{k+r} K_{k}\right) R_{k}^{-1} \Gamma_{k}=R_{k+1} H_{k} R_{k}^{-1} \Gamma_{k} .
\end{aligned}
$$

From Assumption 5, it follows that $R_{k}^{-1} \Gamma_{k}$ is solution of a uniformly exponentially stable system. Hence, there are constants $c_{1}>0$ and $c_{2}>0$ such that for all $k_{0} \geq 1$ and $k \geq k_{0}$, the inequality

$$
\left|\Gamma_{k}\right| \leq c_{1} e^{c_{2}\left(k_{0}-k\right)}\left|\Gamma_{k_{0}}\right|
$$

is satisfied. Recalling that the sequence $K_{k} R_{k}^{-1}$ is bounded, we conclude that there is a constant $c_{3}>0$ such that for all $k_{0} \geq 1$ and $k \geq k_{0}$, the inequality

$$
\left|u_{k}\right| \leq c_{3} e^{c_{2}\left(k_{0}-k\right)}\left|\Gamma_{k_{0}}\right|
$$

is satisfied, namely, $c_{3}=c_{1} \sup _{k}\left|K_{k} R_{k}^{-1}\right|$. Next, observe that (46) gives

$$
x_{k}=R_{k}^{-1}\left(\left(A_{*}^{r}\right)^{-1} \Gamma_{k}-\left(A_{*}^{r}\right)^{-1} \sum_{i=1}^{r} A_{*}^{i-1} G_{k+r-i} u_{k-i}\right) .
$$

Also, the boundedness of the sequences $B_{k}$ and $R_{k}$ gives boundedness of the sequence $G_{k}$. From (50), (51), (52) and the properties of the matrices $R_{k}$, we can conclude.

Remark 3. If we know a strict quadratic Lyapunov function for (43), then, by adapting ideas of [25], we can build a function of Lyapunov-Krasovskii functional type for (35) in closed loop with (44).

\subsection{Important Remark on Discretizations}

Consider a continuous time time-varying system

$$
\dot{x}(t)=M(t) x(t)+N(t) u(t-\tau)
$$

where the delay $\tau>0$ is constant. Assume that $M$ is periodic, and that $M$ and $N$ are continuous. Since we do not assume that a fundamental solution of $\dot{\xi}(t)=M(t) \xi(t)$ is known, no standard stabilization technique available in the literature applies. 
It is natural to approximate the system (53) by

$$
\dot{x}(t)=M\left(t_{k}\right) x(t)+N\left(t_{k}\right) u\left(t_{k-r}\right)
$$

where $t_{k+1}-t_{k}=\nu, \nu>0$ is a sufficiently small constant, and $r>0$ is an integer. By integrating (54), we obtain

$$
x\left(t_{k+1}\right)=e^{\nu M\left(t_{k}\right)} x\left(t_{k}\right)+\left(\int_{t_{k}}^{t_{k+1}} e^{M\left(t_{k}\right)\left(t_{k}+\nu-\ell\right)} \mathrm{d} \ell\right) N\left(t_{k}\right) u\left(t_{k-r}\right) .
$$

That way, we can handle cases which are not covered by the results of our work [24], e.g., because we do not need an upper bound on the allowable delays, nor do we need a formula for the fundamental matrix for $\dot{x}=M(t) x$ to use in the reduction model control. Notice that, to simplify, we can approximate (55) by

$$
x_{k+1}=\left[I_{n}+\nu M\left(t_{k}\right)\right] x_{k}+\nu N\left(t_{k}\right) u_{t_{k-r}} .
$$

If $\nu$ is sufficiently small, then all of the matrices $A_{k}=I_{n}+\nu M\left(t_{k}\right)$ are invertible. Moreover, we can select the sequence $t_{k}$ so that the sequence $A_{k}$ is periodic.

\section{Examples}

\subsection{Continuous Time Example}

Consider the system

$$
\dot{x}(t)=\left(\sin (t)+\frac{1}{2}\right) x(t)+u(t-\tau),
$$

where $x$ and $u$ are scalar valued. It is unstable when the input is set to zero, but Theorem 1 applies to (57). In fact, the fundamental matrix of $\dot{x}=\left(\sin (t)+\frac{1}{2}\right) x$ is

$$
X(t)=e^{\frac{1}{2} t+1} e^{-\cos (t)} .
$$

Therefore, Theorem 1 leads us to consider the change of coordinates (27), which in our case leads to our choice $z=e^{\cos (t)} x$. It yields

$$
\dot{z}=\frac{1}{2} z+e^{\cos (t)} u(t-\tau)
$$

Applying the reduction model approach from Lemma 1, we obtain the undelayed system

$$
\dot{Z}(t)=\frac{1}{2} Z(t)+e^{\cos (t+\tau)-\frac{\tau}{2}} u(t)
$$

where

$$
Z(t)=e^{\cos (t)} x(t)+\int_{t-\tau}^{t} e^{\frac{1}{2}(t-m-\tau)} e^{\cos (m+\tau)} u(m) \mathrm{d} m
$$

This system is asymptotically stabilized by

$$
u(t)=-e^{-\cos (t+\tau)+\frac{\tau}{2}} Z(t)=-e^{-\cos (t+\tau)+\frac{\tau}{2}}\left[e^{\cos (t)} x(t)+\int_{t-\tau}^{t} e^{\frac{1}{2}(t-m-\tau)} e^{\cos (m+\tau)} u(m) \mathrm{d} m\right]
$$

which gives the closed loop system $\dot{Z}(t)=-\frac{1}{2} Z(t)$.

\subsection{Discrete Time Example}

Consider the system (35) with $B_{k}=1$ for all $k$ and with the sequence of real numbers $A_{k}$ defined as follows:

$$
A_{2 l}=2 \text { and } A_{2 l+1}=\frac{2 l^{2}+1}{l^{2}+1} \text { for all } l \geq 0
$$

Observe that this sequence is not periodic. With the notation of Section 4 , we have $P_{0}=1, P_{1}=\frac{1}{2}$, and the following for all $l \geq 1$ :

$$
P_{2 l+1}=\prod_{i=0}^{2 l} A_{i}^{-1}=\frac{1}{2^{l+1}} \prod_{i=0}^{l-1} \frac{i^{2}+1}{2 i^{2}+1} \quad \text { and } \quad P_{2 l}=\prod_{i=0}^{2 l-1} A_{i}^{-1}=P_{2 l+1} A_{2 l}=\frac{1}{2^{l}} \prod_{i=0}^{l-1} \frac{i^{2}+1}{2 i^{2}+1}
$$


Let us choose $A_{*}=2$. Then, for all $l \geq 1$, the functions $R_{k}=A_{*}^{k} P_{k}$ from Assumption 4 are

$$
R_{2 l+1}=2^{2 l+1} \frac{1}{2^{l+1}} \prod_{i=0}^{l-1} \frac{i^{2}+1}{2 i^{2}+1}=2^{l} \prod_{i=0}^{l-1} \frac{i^{2}+1}{2 i^{2}+1}=R_{2 l} .
$$

Consequently, for all $l \geq 1$, we have

$$
\begin{aligned}
0<R_{2 l}=R_{2 l+1} & \leq 2^{l} \prod_{i=0}^{l-1} \frac{i^{2}+1}{2 i^{2}+1}=\exp \left(\sum_{i=0}^{l-1} \ln \left(\frac{2 i^{2}+2}{2 i^{2}+1}\right)\right)=\exp \left(\sum_{i=0}^{l-1} \ln \left(1+\frac{1}{2 i^{2}+1}\right)\right) \\
& \leq \exp \left(\sum_{i=0}^{l-1} \frac{1}{2 i^{2}+1}\right) \leq \exp \left(1+\frac{1}{2} \sum_{i=1}^{\infty} \frac{1}{i^{2}}\right)<\infty
\end{aligned}
$$

and

$$
0<R_{2 l}^{-1}=R_{2 l+1}^{-1} \leq \frac{1}{2^{l}} \prod_{i=0}^{l-1} \frac{2 i^{2}+1}{i^{2}+1} \leq 1 .
$$

Hence, we can satisfy the bound requirement (39) from Assumption 4 with the bound

$$
c=\exp \left(1+\frac{1}{2} \sum_{i=1}^{\infty} \frac{1}{i^{2}}\right)
$$

We now apply Theorem 2 to the corresponding discrete time system (35), with the preceding choices of $A_{k}, B_{k}=1$ for all $k$, and $r=4$. With the choices

$$
K_{k}=-\frac{A_{k}}{R_{k+1}^{-1} R_{k+5}}
$$

the matrices $H_{k}$ from Assumption 5 become

$$
H_{k}=A_{k}+R_{k+1}^{-1} R_{k+5} K_{k}=0,
$$

so Assumption 5 is satisfied. Then Theorem 2 provides us with the stabilizing control law

$$
u_{k}=-16 \frac{A_{k} R_{k+1}}{R_{k+5}} x_{k}-\sum_{i=1}^{4} \frac{A_{k} R_{k+1} R_{k+5-i}}{R_{k} R_{k+5}} 2^{i-1} u_{k-i} .
$$

Using our formulas (65) for the $R_{k}$ 's and (71), we obtain the formulas

$$
\begin{aligned}
u_{2 l}= & -32 \frac{R_{2 l}}{R_{2 l+4}} x_{2 l}-2 \sum_{i=1}^{4} \frac{R_{2 l+5-i}}{R_{2 l+4}} 2^{i-1} u_{2 l-i} \\
= & 2\left(\frac{2(l+1)^{2}+1}{(l+1)^{2}+1}\left\{-2 \frac{2 l^{2}+1}{l^{2}+1}\left(2 x_{2 l}+u_{2 l-4}\right)-u_{2 l-2}-2 u_{2 l-3}\right\}-u_{2 l-1}\right) \text { and } \\
u_{2 l+1}= & -\frac{2 l^{2}+1}{l^{2}+1}\left(16 \frac{R_{2 l+2}}{R_{2 l+6}} x_{2 l+1}+\sum_{i=1}^{4} \frac{R_{2 l+2} R_{2 l+6-i}}{R_{2 l+1} R_{2 l+6}} 2^{i-1} u_{2 l+1-i}\right) \\
= & -\frac{2 l^{2}+1}{l^{2}+1}\left\{4\left(\prod_{i=l+1}^{l+2} \frac{2 i^{2}+1}{i^{2}+1}\right) x_{2 l+1}+\frac{l^{2}+1}{2 l^{2}+1} \frac{2(l+2)^{2}+1}{(l+2)^{2}+1}\left(u_{2 l}+2 u_{2 l-1}\right)\right. \\
& \left.+2 \frac{l^{2}+1}{2 l^{2}+1} \prod_{i=l+1}^{l+2} \frac{2 i^{2}+1}{i^{2}+1}\left(u_{2 l-2}+2 u_{2 l-3}\right)\right\}
\end{aligned}
$$

for all $l$, which ensure the desired exponential stability property from Theorem 2 . 


\section{Conclusions}

We provided new reduction model method input delay compensating controllers for time-varying continuous and discrete time systems, using Floquet's theory and its extensions. Compared with other reduction model methods for time-varying continuous time linear systems (such as our recent work [24]), two potential advantages of our approach are that (a) we do not require closed form expressions for the fundamental matrix for the corresponding uncontrolled drift systems and (b) our new results allow arbitrarily long input delays. In future works, we will investigate stability problems for systems with several and time-varying delays.

\section{References}

[1] T. Anh, L. Hien, and V. Phat. Stability analysis for linear non-autonomous systems with continuously distributed multiple time-varying delays and applications. Acta Mathematica Vietnamica, 36(2):129$143,2011$.

[2] Z. Artstein. Linear systems with delayed controls: a reduction. IEEE Transactions on Automatic Control, 27(4):869-879, 1982.

[3] L. Barreira and C. Valls. Ordinary Differential Equations, Qualitative Theory. American Mathematical Society, Providence, RI, 2012.

[4] N. Bekiaris-Liberis and M. Krstic. Nonlinear Control Under Nonconstant Delays. Society for Industrial and Applied Mathematics, Philadelphia, PA, 2013.

[5] D. Bini, N. Higham, and B. Meini. Algorithms for the matrix pth root. Numerical Algorithms, 39(4):349$378,2005$.

[6] S. Bittanti and P. Colaneri. Companion forms and cyclic matrices for discrete-time periodic systems. In Proceedings of the IFAC 15th Triennial World Congress, pages 1-6, Barcelona, Spain, 2002.

[7] D. Bresch-Pietri, J. Chauvin, and N. Petit. Prediction-based stabilization of linear systems subject to input-dependent input delay of integral type. IEEE Transactions on Automatic Control, 59(9):23852399, 2014.

[8] D. Bresch-Pietri and N. Petit. Robust compensation of a chattering time-varying input delay. In Proceedings of the IEEE Conference on Decision and Control, pages 457-462, Los Angeles, LA, 2014.

[9] M. Cloosterman, N. van de Wouw, W. Heemels, and H. Nijmeijer. Stability of networked control systems with large delays. In Proceedings of the IEEE Conference on Decision and Control, pages 5017-5022, New Orleans, LA, 2007.

[10] W. Culver. On the existence and uniqueness of the real logarithm of a matrix. Proceedings of the American Mathematical Society, 17:1146-1151, 1966.

[11] P. Van Dooren and J. Sreedhar. When is a periodic discrete-time system equivalent to a time-invariant one? Linear Algebra and Its Applications, 131/132:131-151, 1994.

[12] P. Garcia, A. Gonzalez, P. Castillo, R. Lozano, and P. Albertos. Robustness of a discrete-time predictorbased controller for time-varying measurement delay. Control Engineering Practice, 20(2):102-110, 2011.

[13] Y. Hayakawa and T. Jimbo. Floquet transformations for discrete-time systems: Equivalence between periodic systems and time-invariant ones. In Proceedings of the IEEE Conference on Decision and Control, pages 5140-5145, Cancun, Mexico, 2008.

[14] E. Hernandez-Vargas, P. Colaneri, R. Middleton, and F. Blanchini. Discrete-time control for switched positive systems with application to mitigating viral escape. International Journal of Robust and Nonlinear Control, 21(10):1093-1111, 2011.

[15] N. Higham. Functions of Matrices: Theory and Computation. Society for Industrial and Applied Mathematics, Philadelphia, PA, 2008.

[16] I. Karafyllis and M. Krstic. Robust predictor feedback for discrete-time systems with input delays. International Journal of Control, 86(9):1652-1663, 2013. 
[17] M. Krstic. Delay Compensation for Nonlinear, Adaptive, and PDE Systems. Birkhauser, Boston, 2009.

[18] M. Krstic. Input delay compensation for forward complete and feedforward nonlinear systems. IEEE Transactions on Automatic Control, 55(1):287-303, 2010.

[19] W. Kwon and A. Pearson. Feedback stabilization of linear systems with delayed control. IEEE Transactions on Automatic Control, 25(2):266-269, 1980.

[20] M. Malisoff and F. Zhang. Robustness of adaptive control under time delays for three-dimensional curve tracking. SIAM Journal on Control and Optimization, 53(4):2203-2236, 2015.

[21] D. Mayne. Control of linear systems with time delay. Electronics Letters, 4(20):439-440, 1968.

[22] F. Mazenc and M. Malisoff. Local stabilization of nonlinear systems through the reduction model approach. IEEE Transactions on Automatic Control, 59(11):3033-3039, 2014.

[23] F. Mazenc, M. Malisoff, and Z. Lin. Further results on input-to-state stability for nonlinear systems with delayed feedbacks. Automatica, 44(9):2415-2421, 2008.

[24] F. Mazenc, M. Malisoff, and S-I. Niculescu. Reduction model approach for linear time-varying systems with delays. IEEE Transactions on Automatic Control, 59(8):2068-2082, 2014.

[25] F. Mazenc, S-I. Niculescu, and M. Krstic. Lyapunov-Krasovskii functionals and application to input delay compensation for linear time-invariant systems. Automatica, 48(7):1317-1323, 2012.

[26] L. Perko. Differential Equations and Dynamical Systems. Springer-Verlag, New York, NY, 2001.

[27] V. Phat and L. Hien. An application of Razumikhin theorem to exponential stability for linear nonautonomous systems with time-varying delay. Applied Mathematics Letters, 22(9):1412-1417, 2009.

[28] J. Sreedhar and P. Van Dooren. Discrete-time periodic systems: a Floquet approach. In Proceedings of the Conference on Information Sciences and Systems, pages 194-195, Baltimore, MD, 1993.

[29] S. Tiwari and Y. Wang. On stability properties of time-varying systems with delays. In Proceedings of the IEEE Conference on Decision and Control and European Control Conference, pages 314-319, Orlando, FL, 2011.

[30] E. Witrant, C. Canudas de Wit, D. Georges, and M. Alamir. Remote stabilization via communication networks with a distributed control law. IEEE Transactions on Automatic Control, 52(8):1480-1485, 2007. 